\title{
Imaging and Modeling the Dynamics of Clathrin-Mediated Endocytosis
}

\author{
Marcel Mettlen ${ }^{1}$ and Gaudenz Danuser ${ }^{2}$ \\ ${ }^{1}$ Department of Cell Biology, University of Texas Southwestern Medical Center, Dallas, Texas 75235-9039 \\ ${ }^{2}$ Department of Cell Biology, Harvard Medical School, Boston, Massachusetts 02115 \\ Correspondence: gaudenz_danuser@hms.harvard.edu
}

Clathrin-mediated endocytosis (CME) plays a central role in cellular homeostasis and is mediated by clathrin-coated pits (CCPs). Live-cell imaging has revealed a remarkable heterogeneity in CCPassembly kinetics, which can be used as an intrinsic source of mechanistic information on CCP regulation but also poses several major problems for unbiased analysis of CME dynamics. The backbone of unveiling the molecular control of CME is an imagingbased inventory of the full diversity of individual CCP behaviors, which requires detection and tracking of structural fiduciaries and regulatory proteins with an accuracy of $>99.9 \%$, despite very low signals. This level of confidence can only be achieved by combining appropriate imaging modalities with self-diagnostic computational algorithms for image analysis and data mining.

$\mathrm{C}$ lathrin-mediated endocytosis (CME) drives the uptake of diverse receptor-bound macromolecules and is one of the main endocytic mechanisms constitutively active in all mammalian cells. Clathrin-coated vesicles (CCVs) were the first transport vesicles to be isolated (Pearse 1975), which subsequently led to the identification of clathrin and the heterotetrameric adaptor protein AP2 as the major coat components (Pearse 1976, 1978). Further research in this area was spurred by the discovery that familial hypercholesterolemia is caused by a single substitution of a cysteine for a tyrosine in the cytoplasmic tail of the low-density lipoprotein receptor (LDLR), which disrupts its endocytic internalization motif and prevents its concentration in clathrin-coated pits (CCPs) (Anderson et al.
1977). In the following decades, biochemistry combined with molecular biology and electron microscopy (EM) have revealed much about the molecular players involved in CME (reviewed by Conner and Schmid 2003; Schmid and McMahon 2007; McMahon and Boucrot 2011; Boettner et al. 2012). Today, we know that CME is initiated via assembly of clathrin and AP2 to form CCPs and that receptor-ligand complexes (referred to as "cargo") are concentrated in CCPs via direct interactions between endocytic motifs within their cytoplasmic domains and adaptor molecules that recruit clathrin. With the aid of a multitude of endocytic accessory proteins (EAPs) - many with as-yet poorly defined functions-CCPs undergo stabilization, maturation, and invagination. Finally, membrane fis-

Editors: Sandra L. Schmid, Alexander Sorkin, and Marino Zerial

Additional Perspectives on Endocytosis available at www.cshperspectives.org

Copyright (C) 2014 Cold Spring Harbor Laboratory Press; all rights reserved; doi: 10.1101/cshperspect.a017038

Cite this article as Cold Spring Harb Perspect Biol 2014;6:a017038 
sion, catalyzed by the GTPase dynamin, pinches off the CCV carrying its cargo into the cell.

Although powerful and invaluable, bulk biochemical assays can only report cumulative and ensemble-averaged effects on CME, whereas EM only provides static snapshots of highly dynamic structures. Both approaches are not sufficient to resolve critical, rate-limiting stages of CCP maturation and alternative outcomes that prevent CCV internalization. They are also not sufficient to probe the frequently overlapping functions of individual components in CCP formation and maturation. Perturbation of molecular players in a system with such redundancy may lead to no detectable shifts, or to detectable shifts that merely represent system adaptation, and thus may not reveal the actual function of the targeted component itself. Moreover, perturbing CME may globally interfere with cell homeostasis, which can also elicit phenotypes unrelated to the actual functions of the target. To remedy these issues, it is necessary to follow the dynamics of CME at the level of individual CCPs and to correlate these behaviors with differential patterns of cargo and EAP recruitment and activity.

These goals became approachable with the "GFP revolution" in the 1990s, which was paralleled by leaps in the sensitivity of digital light microscopy. For CME, the power of these technologies was first shown by Keen and colleagues, who used a green fluorescent protein (GFP) fusion of the clathrin light chain (CLC) to image clathrin dynamics by time-lapse wide-field epifluorescence microscopy (Gaidarov et al. 1999). Since then, numerous live-cell imaging studies have revealed remarkable heterogeneity in CCP assembly kinetics and internalization (Rappoport and Simon 2003; Ehrlich et al. 2004; Keyel et al. 2004; Merrifield et al. 2005; Loerke et al. 2009; Taylor et al. 2011). Although the physiological and molecular bases for this heterogeneity remain to be uncovered, the working hypothesis is that CCP heterogeneity arises from variations in molecular composition, in cortical membrane mechanics, and in differences between cell types. More recent advances in imaging and computational image data analyses have made it possible to determine the order in which
EAPs are incorporated or released from growing CCPs. Thus, multidimensional live-cell imaging and mathematical models, in combination with very mild chemical, molecular, and mechanical perturbations, may uncover how the molecular composition of an assembling CCP affects its behavior. In the following we describe the developments of imaging modalities and image analysis methods that have led to the current state of the art in quantitative imaging of CME.

\section{THE QUEST FOR ACCURACY IN IMAGING AND ANALYSIS - OVERVIEW OF APPROACHES}

Determining the functional hierarchy of EAPs in control of CME dynamics requires (1) an accurate definition of the initiation of a CCP, (2) a robust tracking of the nascent and then growing structure over time, and (3) an accurate definition of the invagination and internalization of stabilized CCPs as CCVs, or of the disassembly of unstable CCPs as abortive structures. Differences in the molecular composition and/or assembly kinetics between stable and unstable CCPs may then reveal essential functions of EAPs. Unfortunately, these measurement tasks rely on solving a notoriously unforgiving problem: how to determine the lifetime of a macromolecular assembly based on an image signalmost often fluorescence-emitted by one subcomponent. Importantly, any error in identification and tracking causes a systematic underestimation of the lifetime. This problem is illustrated by simulating the life spans of an assembly and the influence of a small fraction of random tracking misses on its lifetime distribution (Fig. 1A). With a probability of only one miss in 200 frames, the lifetime distribution is already significantly distorted; and with two misses in 100 frames (i.e., signal tracking with 98\% confidence), the original Rayleigh distribution used to generate the ground truth traces is converted into an exponential lifetime distribution, in which traces with apparently short lifetimes dominate. In most tracking applications, the undesired dominance of short traces in lifetime statistics is alleviated by excluding structures with a lifetime below a certain thresh- 


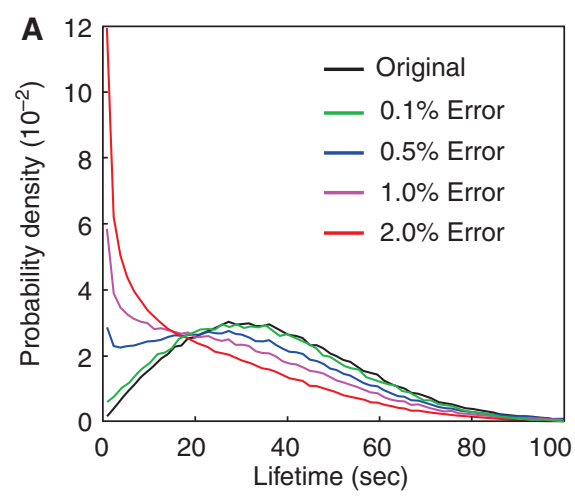

B

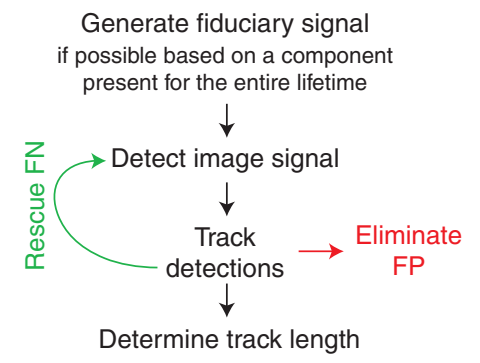

Figure 1. Tracking macromolecular assemblies. $(A)$ The measurement of lifetime distributions is notoriously susceptible to tracking errors, as illustrated by the influence of a small fraction of tracking misses on the lifetime curve of 100,000 simulated trajectories. (B) Flowchart of track length determination. FN, False negative; FP, false positive.

old. However, this approach is inappropriate as it is generally unknown which threshold will produce inferred population statistics closest to the true behavior. Furthermore, lifetimes above the threshold are also contaminated by tracking misses. Thus, lifetime statistics of macromolecular assemblies are difficult to compare between studies, and even within the same studies, as the setting of a threshold introduces an uncontrolled level of arbitrariness, unless the tracking confidence reaches the range of $99.9 \%$.

The quest for high-confidence tracking translates into quests for high accuracy in three steps before determination of the track length (Fig. 1B). First, a fiduciary component has to be identified that marks the beginning of the assembly process and remains associated with the structure until it disappears. Second, the marker images must be detected. This requires
Dynamics of Clathrin-Mediated Endocytosis

a marker signal that is statistically higher than noise. Translating the above requirement for $>99.9 \%$ confidence into the detection problem, marker signals would have to be detected as outliers to the background image distribution with a $p$ value $<0.001$. The first step toward this goal is the implementation of imaging modalities that capture with high sensitivity the signal of a fluorescent fiduciary molecule while minimizing the signal from unspecific background, including detector noise. Even with the best currently available imaging strategies, it is impossible to generate marker images that are distinguishable from other image signals at a confidence level of $>99.9 \%$, especially at the beginning of a CCP trace in which molecular components are low in abundance. Therefore, to achieve the required tracking confidence, it is necessary to amplify the marker signal and oppress noise in a postacquisition step. Third, the detected signals in individual frames have to be connected in time (i.e., tracked). At the beginning of a trace, a new signal has to be connected to corresponding signals in later time points, without connecting it to signals from other CCPs in preceding time points. The inverse applies to the termination of a trace. Moreover, the signal detector will produce false positive (FP) and false negative (FN) detections, of which the probabilities of occurrence are in an inverse relationship. During tracking, these errors can be eliminated. When carefully balanced and calibrated, the combination of the three steps makes it possible to meet the strict requirements in terms of tracking accuracy. In the following, we review reported approaches toward implementing these steps and provide some critical evaluations of remaining stumbling blocks.

\section{DEFINITION OF FIDUCIARIES}

The first task in live-imaging CME was the definition of molecular fiduciaries that faithfully visualize the initiation, maturation, and internalization of CCPs (Fig. 2A). These markers turned out to be different in different model organisms.

In mammalian cells, fluorescently labeled CLCs and small AP2 subunits are obvious 
M. Mettlen and G. Danuser

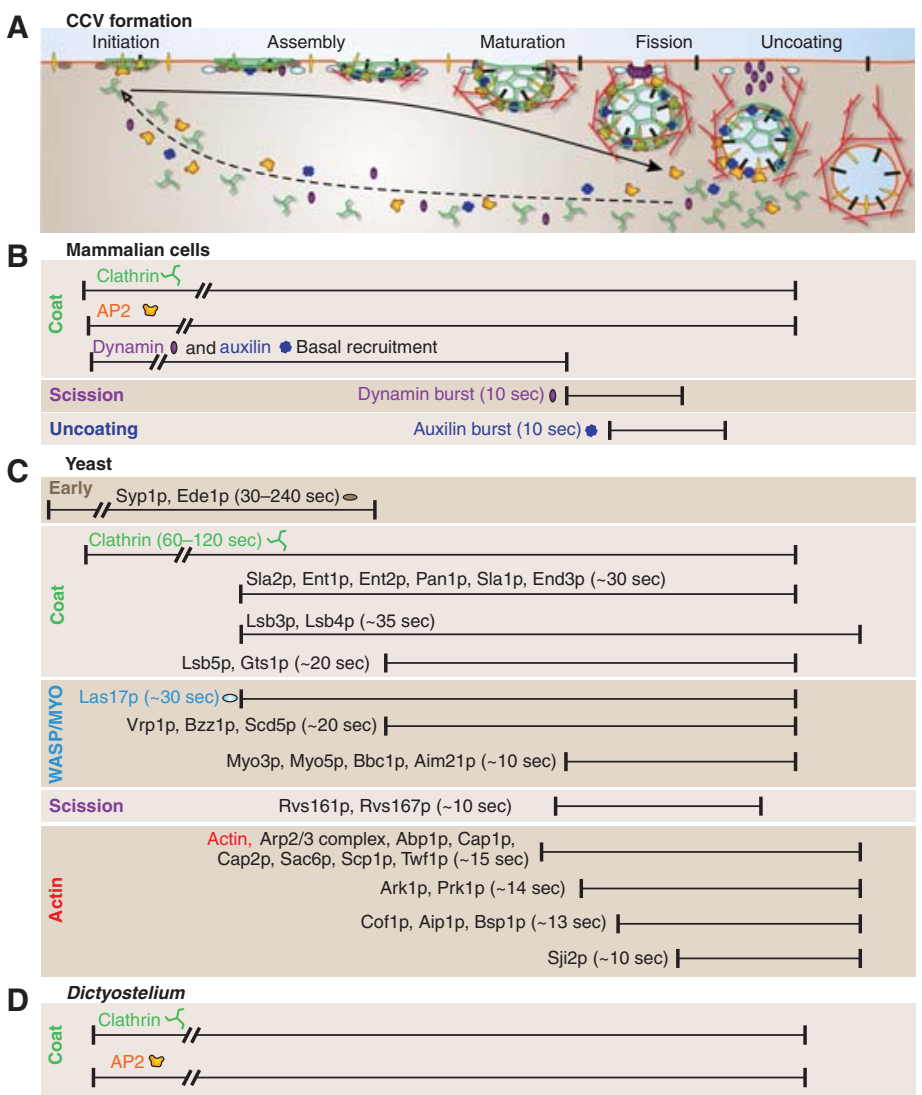

Figure 2. Fiduciaries in different model systems. (A) Formation of clathrin-coated vesicles (CCVs) is a multistep process, starting with the initiation, assembly, and maturation of CCPs, followed by scission from the plasma membrane and uncoating of the freshly formed CCV. To visualize these steps, various fiduciaries can be used in (B) mammalian cells, $(C)$ yeast, and (D) Dictyostelium. (Figure created from data in Kaksonen et al. 2006, Loerke et al. 2009, Taylor et al. 2011, and Macro et al. 2012.)

choices for fiduciaries, as their respective protein assemblies are the most abundant coat components and present throughout the entire lifetime of a CCP (Fig. 2A,B). Until recently, all studies in mammalian cells had to rely on overexpression of labeled markers. Although shifts in the relative abundance of components of fairly small macromolecular structures like the clathrin coat may be disruptive to the assembly process, the earliest imaging studies using GFP fusions to CLC and AP2 subunits established that these markers preserve the stoichiometry of clathrin triskelions and adaptors and do not interfere with CME function or dynamics (Gaidarov et al. 1999; Ehrlich et al. 2004; Merrifield et al. 2005; Loerke et al. 2009). More recently, this premise has been challenged by a study that introduced labeling of fiduciaries through direct genomic tagging, allowing the expression of fusion proteins under the endogenous promoter (Doyon et al. 2011). Although elegant, this approach turned out to be insufficient for identifying complete CCP trajectories as the substoichiometric incorporation of CLCs in the triskelia lead to low signal-to-noise ratios (SNR) and thus erroneous tracking (Aguet et al. 2013). Genome editing, however, might be the method of choice to study EAPs that play a more direct regulatory and/or structural role, without perturbing their normal dynamic behavior. Given a robust fiduciary of the lifetime of a CCP, extremely low signals derived from endogenously 
Dynamics of Clathrin-Mediated Endocytosis

tagged EAPs can be detected with high sensitivity using a master/slave approach, in which the fiduciary signal defines time and location of the signal readout in the low SNR slave channel (Aguet et al. 2013).

Because of the relative ease of genetic manipulations, Saccharomyces cerevisiae and Schizosaccharomyces pombe have been invaluable model systems for the study of CME. Over the past decades more than 50 proteins have been implicated in the assembly and internalization of CCPs (Engqvist-Goldstein and Drubin 2003). Although there are very strong similarities between yeast and mammals, there are also marked differences (see Merrifield and Kaksonen 2014). For instance, in yeast, a functional connection between actin and endocytosis has been firmly established (Newpher et al. 2005; Sirotkin et al. 2005; Kaksonen et al. 2006; Aghamohammadzadeh and Ayscough 2009), whereas in mammalian cells, CME is largely actin independent with actin polymerization only required under certain circumstances (Boulant et al. 2011; see also Mooren et al. 2012). Conversely, the critical endocytic core machinery in higher metazoans, composed of AP2, dynamin, and clathrin, is dispensable in yeast (Tan et al. 1993; Huang et al. 1999; Mitsunari et al. 2005; Carroll et al. 2009; Smaczynska-de et al. 2010). Nonetheless, based on the stereotypical hierarchy of protein recruitment to endocytic sites, tracking CCP dynamics in this system was achieved by following temporally overlapping molecular modules that span the entire life span of a CCP from coat assembly to vesicle scission (Fig. 2C) (Kaksonen et al. 2003, 2005, 2006; Huckaba et al. 2004; Newpher et al. 2005).

An alternative model with similarly powerful genetic tools, yet in some ways stronger similarities to higher eukaryotes than yeast, is Dictyostelium discoideum (Fig. 2D). As in yeast, actin is required for CME (Brady et al. 2010), whereas AP2 is dispensable (Macro et al. 2012). However, in contrast to yeast but similar to higher eukaryotic cells, micropinocytosis requires clathrin (O'Halloran and Anderson 1992; Ruscetti et al. 1994) and only recently, a bona fide cargo that requires clathrin for internalization has been identified (Macro et al. 2012).

\section{CHOICE OF IMAGING MODALITY}

The second task in imaging CME is the acquisition of time-lapse fluorescence image sequences that will enable subsequent image tracking of CCPs. Careful considerations have to be made with regard to the choices of cell culture, imaging conditions, spatiotemporal sampling, etc. Owing to space limitations, we focus here on the selection of an appropriate imaging modality. Two properties of CCPs drive this process: (1) so-called canonical CCPs are small (i.e., less than the wavelength of visible light $[\sim 500 \mathrm{~nm}])$, and (2) only a small fraction of the fluorescently labeled CCP components are incorporated in CCPs, whereas the remainder is either diffuse in the cytoplasm or bound to other vesicular structures. Property (1) requires diffraction-limited imaging, whereas property (2) requires a narrow optical section that limits the acquisition of fluorescence signal to the plasma membrane. Most commonly, both conditions have been satisfied together by using either total internal reflection fluorescence microscopy (TIRFM) (Toomre and Manstein 2001) or confocal microscopy (Maddox et al. 2003).

In TIRFM, the sample is illuminated by a fluorescence excitation beam at a very oblique angle, such that the light is completely reflected at the top surface of the glass slide. This creates an evanescent field of light that penetrates into the sample with an exponentially decaying energy and a relevant penetration depth of $\sim 100 \mathrm{~nm}$. Accordingly, this mode of fluorescence imaging is exquisitely well suited to selectively illuminate fluorescent probes at the ventral plasma membrane of adherent cells cultured on glass, including those marking CCP components. Indeed, the majority of publications on imaging CME has used this imaging modality as the resolution of individual molecular steps in CME falls exclusively within its application area (e.g., Rappoport and Simon 2003; Keyel et al. 2004; Merrifield et al. 2005; Loerke et al. 2009; Mattheyses et al. 2011; Carroll et al. 2012) and recent studies have shown that the limits in sensitivity of TIRFM can be pushed to single-molecule detection of CCP components (Bocking et al. 2011; Cocucci et al. 2012). Despite these 
overwhelming advantages, TIRFM also has significant drawbacks. First, the approach relies on flat cells spread on cover glass. Nonadherent cells like yeasts are thus less well suited, although TIRF imaging has been accomplished by squeezing cells (Kaksonen et al. 2005; Burston et al. 2009). Second, it is well appreciated that cells on glass are often hyperadherent and that protein expression and activity is significantly distorted (Pampaloni et al. 2010), which questions the physiological relevance of TIRFM-based analyses of CME. Third, endocytosis occurs also on nonadherent cell surfaces. We understand much less of the functioning of the endocytic machinery under these conditions because imaging single CCPs is more difficult. It has been shown that in cells selected for very low expression of a fluorescent fiduciary, the optical sectioning of regular wide-field Epi-fluorescence imaging by high-resolution objective lenses, when focused at the thin edges of adherent cells, can be sufficient to produce a punctate signal of the fiduciary associated with CCPs that is distinct from the diffuse background signal of dissociated fiduciary in the cytoplasm (Gaidarov et al. 1999). However, the large axial extension of the Epi-imaging volume requires additional and potentially arbitrary criteria for distinguishing puncta at the plasma membrane, that is, CCPs, from puncta in the cytoplasm (i.e., $\mathrm{CCVs}$, etc.). Moreover, the requirement for low expression comes at the risk that only a subset of particularly large structures with a high enough number of fluorescent fiduciary can be detected, precluding the unbiased study of CCP dynamics.

The disadvantages of wide-field epifluorescence imaging can be alleviated by using confocal microscopy. Like TIRFM, confocal microscopy narrows the imaging volume, however without restriction to the glass-cell interface. For live-cell imaging of dynamic processes, spinning disk confocal microscopy is the preferred mode of confocal imaging, as it permits fast enough acquisition rates to follow the dynamics of molecular machineries with second scale time sampling (e.g., Ehrlich et al. 2004; Massol et al. 2006). More recently, Kirchhausen and colleagues showed the use of spinning disk confocal microscopy to compare CME dy- namics between the adherent, basolateral and the free, apical surfaces in epithelial monolayers (Boulant et al. 2011). The key limitation of any confocal approach is the exclusion of useful photons by the pinholes, which results in dimmer signals. This precludes the study of EAPs in low abundance, let alone of dynamics at the single-molecule level as achievable by TIRFM. A second drawback of confocal vis-à-vis TIRFM imaging is that, although the detection volume is narrowed, the excitation light path illuminates the entire sample. As a result, the sample tends to bleach faster and phototoxicity can become an issue. For these two reasons, TIRFM has become the preferred imaging modality in molecularly detailed analyses of CME.

\section{Tracking CME Dynamics}

Image time-lapse sequences of CME, especially in mammalian cells, contain thousands of punctate signals of assembling, maturating, and internalizing CCPs. Already by visual inspection of these movies it is evident that CCPs display a wide range of dynamics. This poses three questions: (1) How long do movies need to be, and how fast do they need to be sampled to capture the full spectrum of lifetimes? (2) How many CCPs does one have to track to obtain a representative sample of CCP behavior? (3) What selection criteria for tracking permit the capture of all essential events that control CCP behavior? Although question (1) relates again to the choice of the imaging modality and the optimization of image acquisition parameters that guarantee the capturing of as many frames as possible at minimal bleaching and phototoxicity, questions (2) and (3) relate to the processing of the acquired image data to extract from it relevant statistical representations of CCP behavior. Clearly, a manual measurement of CCP tracks is impossible for all visible events. When forced to select a subset of events, a biased focus on a particular behavior is unavoidable. Accordingly, with the very first imaging studies of CME, researchers made an effort to use semiautomated and automated computer software to detect and track CCP dynamics (Gaidarov et al. 1999; Rappoport and Simon 2003; Ehrlich et al. 2004; Merrifield 
Dynamics of Clathrin-Mediated Endocytosis

et al. 2005; Mattheyses et al. 2011). In particular, the study by (Ehrlich et al. 2004) illustrates early applications of particle tracking to extract representative distributions of CCP lifetimes. But even with such tools at hand, significant dangers of introducing bias remain. First, not all commercial and academic software packages available for particle tracking are suitable for the task of measuring CCP dynamics. Although they all generate some trajectories, it is exceedingly difficult to judge whether the trajectories are actually correct. Visual inspection may not be the best approach for validation, as it suffers from the intrinsic selection biases discussed above (Danuser 2011). Second, software packages require user input of parameters that control the decision making in detection and tracking. Thus, software-based image measurements, although more reproducible, are not necessarily more objective. To make image analyses objective, algorithms should be controlled only by statistical tests allowing the definition of one global confidence level based on which all decisions are made. Moreover, algorithms should be self-diagnostic (i.e., the computational processes recognize variation in data quality and possibly adjust procedures, or provide the operator with indicators of data limitations).

Over the past 10 years, our laboratories have directed efforts toward achieving these goals in software-based analysis of CCP dynamics. In our most recent publication we introduced a model-based particle detection method that identifies diffraction-limited images of CCPs based on a self-trained model of image noise (Aguet et al. 2013). By fitting a model image of a diffraction-limited particle to a putative CCP image, this method can make use of the residuals of this fit and directly compare the uncertainty of the particle brightness estimate to the uncertainty of the local noise threshold. Thus, contrary to other particle detection methods applied in the field, which operate on a fixed threshold of particle brightness alone (e.g., Ehrlich et al. 2004), higher spatial moments of the brightness distribution (e.g., Sbalzarini and Koumoutsakos 2005), or on a more statistically motivated threshold of the ratio between signal brightness and noise (e.g., Rink et al. 2005), this detection method provides a more objective means of diffraction-limited particle detection. Moreover, this procedure is not only adaptive to variable levels of image and background noise, but it significantly increases the sensitivity of the particle detection over alternative methods at essentially zero-rate false discovery (Fig. 3A).

The role of the tracking module is to link the images of the same CCP between consecutive frames. Compared to other problems in particle tracking (e.g., of fast-moving and heterogeneous surface receptors) (Jaqaman et al. 2011), following CCPs is relatively simple given their fairly constricted movement. However, in CME, complexity in tracking arises from the continuous appearance and disappearance of CCP images and from the possibility that CCP signals, especially at early stages, may not be strong enough to be robustly detected in every single frame. Thus, the tracker needs to be equipped with functionality for so-called gap closing (i.e., links may reach over frames with detection misses). Gap closing must be balanced against bona fide termination and initiation of CCPs. We have proposed tracking algorithms that resolve these competing criteria by iterative global optimization (Jaqaman et al. 2008). "Global" means that decisions on whether two CCP images should be linked over one or multiple putative detection misses are not made CCP by CCP, but by simultaneously considering all plausible scenarios of linking CCP images and selection of the overall most probable solution. The iteration in optimization serves again the self-learning of the algorithm. After a first round, the system computes the distribution of frequency and durations of gaps-noisier images will contain more and longer gaps than less noisy onesand reestimates from these statistics the likelihood for each of the considered scenarios. In addition, the system includes a postprocessing step, in which the image signal in gap locations is reanalyzed. True gaps contain a weak CCP image signal that falls below the confidence level of the detector module, but the signal is still significantly stronger than the background signals detected before CCP initiation or after CCP internalization (Fig. 3B,C). If a gap contains no such weak image signal, the algorithm classifies 
M. Mettlen and G. Danuser
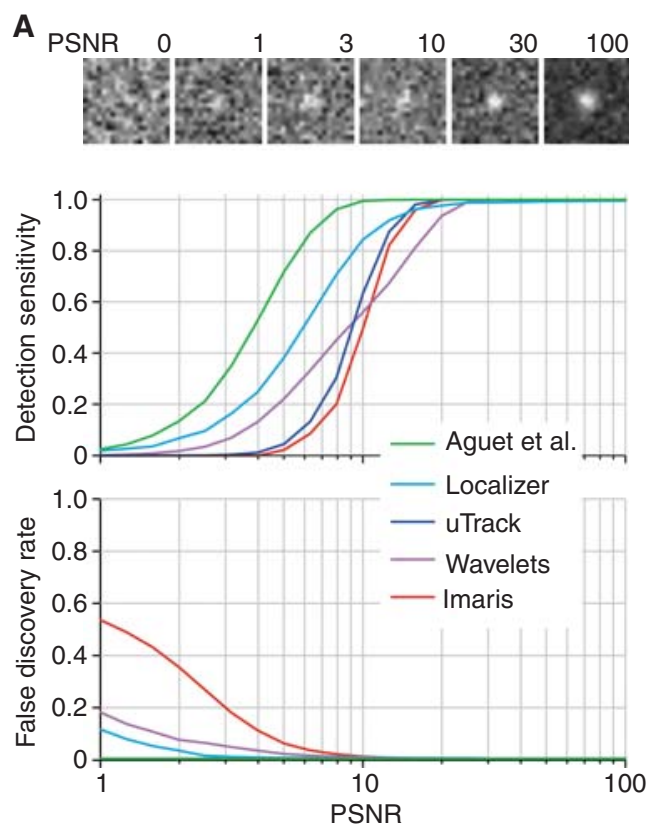

B
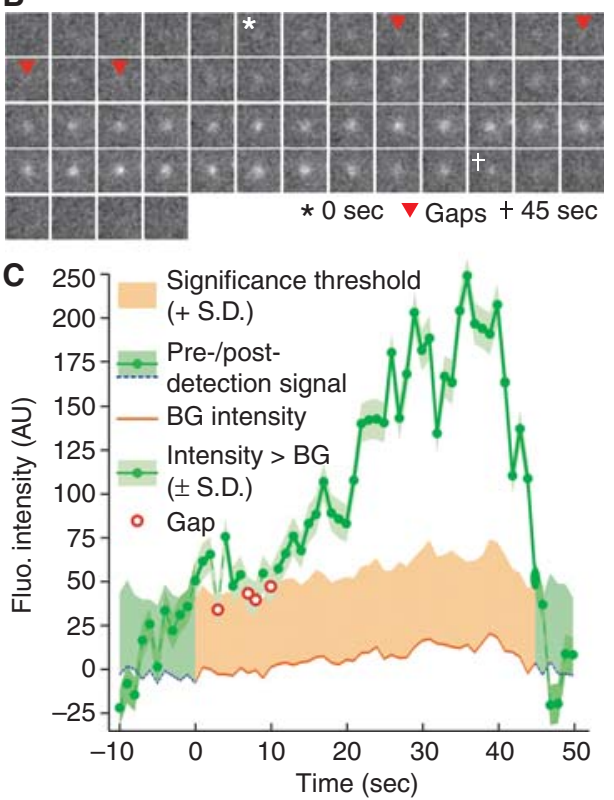

Figure 3. (A) Comparison of detection performance between several state-of-the-art methods (see the Aguet et al. 2013 supplement for a detailed key to the tested methods). Performance was assessed on simulated noisy data containing Gaussian-shaped signals of varying amplitude with additive white Gaussian noise, corresponding to a peak signal-to-noise ratio (PSNR) ranging from 1 to 100. (B) Representative frames and intensity trace of a CCP identified by model-based detection and tracking of eGFP-CLCa. Gaps are defined as frames missed by the detection but recovered during tracking. Uncertainties for the detected intensities (shown as S.D., lightly shaded green band), the significance threshold ( $\sim 2$ S.D. above background noise, orange shaded area), and residual signals in the darkly shaded green regions preceding and following the first and last detected CCP signals, respectively, were determined as described in Aguet et al. (2013). BG, Background; fluo., fluorescence; S.D., standard deviation.

the gap as a false positive and breaks the trajectory into two. This analysis typically results in thousands of intensity traces documenting the accumulation of fluorescent fiduciary at the CCP. With the increasing sensitivity of image acquisition and signal detection, the tracking module tends to capture also a large number of trajectories related to nonfunctional fragments containing fluorescent fiduciaries. Moreover, the cytoplasm is packed with vesicles that may also carry fiduciaries. When these structures enter the imaging volume of a TIRF or confocal microscope as so-called visitors, they generate an image signal that is difficult to distinguish from the signal of bona fide CCPs. Many studies have tried to eliminate the contribution of such trajectories based on the assumption that they are short-lived. Accordingly, all trajectories with a lifetime below a certain threshold are discarded. Not only is this assumption too simplistic - the lifetime distributions of fragments and short-lived CCPs overlap significantly — the choice of a lifetime threshold is arbitrary, which compromises the accurate analysis of early events in CCP maturation. Our most recent addition to computer-based CCP tracking incorporates algorithms that distinguish fragments from bona fide CCPs based on a self-trained classification of the kinetics of fiduciary recruitment (Aguet et al. 2013). Underlying the algorithm is the model that coat fragments and visitors have instantaneous image appearances and disappearances, whereas the coat of a bona fide CCP undergoes a regulated assembly process. Based on this analysis, we found that a large percentage of all detectable 
Dynamics of Clathrin-Mediated Endocytosis

eGFP-CLCa signals are related to fragments and visitors. Notably, a significant fraction of those exceeds the lifetime of $10-12$ sec often used as a cutoff to eliminate short-lived structures.

In conclusion, we posit that manual and semiautomatic strategies introduce too much bias, and that even with automated software solutions, the manual setting of control parameters can cause irreproducibility. We propose global optimization and self-learning strategies as the best way forward to achieve the reproducibility in data analysis required for meaningful discovery of the regulatory mechanisms of CME. Of note, although the frameworks in (Jaqaman et al. 2008; Aguet et al. 2013) have been designed to achieve self-learning and global optimality with an eye toward the particularities of live-cell tracking of single molecules and macromolecular assemblies like CCPs, there are numerous other particle tracking methods that achieve similar goals with slightly modified algorithmic approaches. We refer to a recent review by Meijering et al. (2012) that had been written with the intention of introducing the cell biologist to the vast literature on particle tracking methods in other fields. How well these methods fare in quantifying the dynamics of CME has yet to be determined.

\section{DATA MINING-ANALYSIS OF THE FUNCTION OF EAPS BY MODELING AND PERTURBATION OF THE CCP LIFETIME DISTRIBUTION}

Two of the first imaging studies aiming at a representative sampling of CCP lifetimes revealed broad distributions with a large fraction of the population living $<20 \mathrm{sec}$ but also a significant fraction living for $>90 \mathrm{sec}$ (Fig. 4A) (Ehrlich et al. 2004; Loerke et al. 2009). Using the same mammalian BSC-1 cell model, both studies also agreed on the mean lifetime of $\sim 40 \mathrm{sec}$ and in the observation of a characteristic mode of preferred lifetime between 20 and $30 \mathrm{sec}$. This suggested that $\mathrm{CME}$ is a regulated process with a major decision in the first $30 \mathrm{sec}$ as to whether
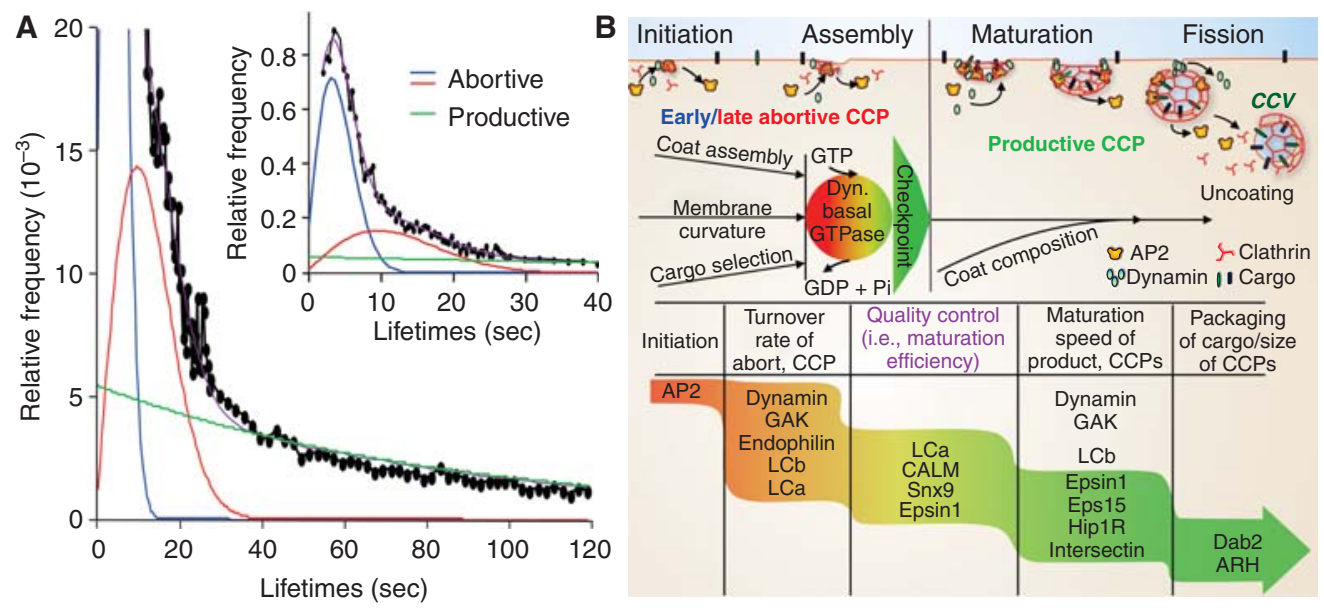

Figure 4. (A) CCP dynamics ( $n=43,568$ CCPs, black curve) were fitted by minimization of the Bayesian information criterion (BIC). This identified three CCP subpopulations, termed early abortive (blue), late abortive (red), and productive CCPs (green; Loerke et al. 2009). (B) By measuring the lifetime distribution of these subpopulations and their relative contributions upon siRNA-mediated depletion of endocytic accessory proteins, we proposed that CCVs are formed from productive CCPs through a maturation process gated by an endocytic checkpoint. CCPs that do not progress beyond this checkpoint abort. Proteins are listed and grouped according to their depletion effects on (1) pit density (column 1); (2) turnover rate of early and late abortive species (column 2); (3) maturation efficiency (i.e., proportion of productive CCPs) (column 3); and (4) maturation speed of productive CCPs (column 4), which provides insight into their respective functions and temporal hierarchy of activities. Dyn., Dynamin. 
CCPs terminate early or continue to live for up to 2-3 min. Importantly, this lifetime distribution stands in stark contrast to a distribution with an exponential decay (Fig. 1A), which would either represent an erroneous measurement, or suggest that CCP internalization after assembly is a random process following firstorder kinetics.

To further understand the mechanisms leading to such lifetimes, Loerke et al. (2009) performed a statistical decomposition of the distribution. Using the Bayesian information criterion for model selection (Schwarz 1978), they found that experimental distributions of CCP lifetimes are best represented by a superposition of two short-lived, Rayleigh distributed populations with modes peaking at $\sim 6 \mathrm{sec}$ and $\sim 12 \mathrm{sec}$ (and with 99\% quantiles of $<15 \mathrm{sec}$ and $<38 \mathrm{sec}$ ) (Fig. 4A, red and blue lines, respectively), and an exponentially decaying population with a $99 \%$ quantile $<140 \mathrm{sec}$ (Fig. 4A, green line). They further showed that the two short-lived populations were likely abortive CCPs undergoing coat disassembly at the plasma membrane, whereas the third more long-lived population constituted productive internalization events and that population size and lifetime of each population were nearly orthogonal parameters. This discovery had two important implications: First, it led to the hypothesis that the rate-limiting steps in the abortive populations are related to a checkpoint system that monitors the progression of CCP assembly and cargo loading. Interestingly, the exponentially decaying lifetime distribution of the productive population suggests that once a CCP has passed beyond the checkpoint, internalization itself is a random event that occurs with a characteristic first-order rate constant (Loerke et al. 2009). Second, the orthogonality of the parameters permits a selective assignment of defects in CME to either early or late stages, as exploited by Mettlen et al. in a depletion screen of EAPs (Fig. 4B) (Mettlen et al. 2009). Hence, acquisition and decomposition of lifetime distributions from a complete tracking of CCPs offers a handle of unprecedented sensitivity into the functional hierarchy among the diverse molecular factors controlling CME.

\section{DIRECT IMAGING OF ENDOCYTIC ACCESSORY PROTEIN ASSOCIATION}

Complementary to defining the functional hierarchy among EAPs by perturbation and lifetime analysis, several studies have used multispectral imaging of fluorescently tagged EAPs to determine the sequence of EAP association with CCP. Although localization is clearly not synonymous for EAP function, this approach is more direct than inference via lifetime decomposition. As simple as the concept of imaging EAP recruitment to CCPs is, there are several challenges to overcome. First, most EAPs operate in a potentially sensitive stoichiometry with other EAPs. Expression of a fluorescent EAP fusion in addition to the endogenous protein pool can severely distort the normal progression of CCP assembly and thus the observed timing of protein recruitment may be entirely irrelevant. Therefore, the possibility of replacing endogenous unlabeled EAP with a labeled fluorescent version has made yeast the preferred model system for such recruitment studies (e.g., Kaksonen et al. 2005; Sirotkin et al. 2010). In mammalian cells, a recent study has begun to address the expression issue by imaging fluorescent EAPs after full substitution of the endogenous EAP for a fluorescent version using genome editing (Doyon et al. 2011). The second challenge arises with the low abundance of EAPs. Especially when imaged at endogenous expression levels, EAPs associate in single-digit copy numbers. Accordingly, the image signals are close to background and often fluctuate significantly because of rapid protein turnover. Robust determination of the temporal and kinetic characteristics of the recruitment therefore requires averaging more than thousands of CCPs. To this end, yeast again offers great advantages because of its reportedly stereotypical protein recruitment. The lifetime of yeast CCPs is fairly constant, allowing straightforward alignment and averaging of EAP intensity traces from multiple CCPs. Such analysis led to the proposal that in yeast endocytosis, EAPs are recruited in distinct modules (Kaksonen et al. 2005). In mammalian cells, however, the wide variation among CCP lifetimes requires more sophisticated alignment 
procedures. The simplest approach would scale EAP traces of different duration to a normalized lifetime value. However, this method blurs recruitment events that occur at a fixed time point in the progression of CCP assembly and maturation. To test the model of a modular EAP recruitment also in mammalian cells, Merrifield and colleagues instead used the strategy of aligning EAP intensity traces relative to the internalization event (Taylor et al. 2011). Although this produced a precise picture of the recruitment of factors that are specifically associated with the scission machinery, EAP recruitment at earlier time points were increasingly blurred by the variable lengths of averaged traces. Although the investigators were able to detect some stereotypical recruitment patterns, these data lack information of the recruitment hierarchy at early time points and in CCPs that prematurely disassemble without a scission event. Similarly, by alignment of traces relative to the CCP initiation event, Kirchhausen and colleagues depicted the hierarchy of protein recruitment during the first $5 \mathrm{sec}$ with single-molecule sensitivity (Cocucci et al. 2012).

Owing to the complications in trace alignment and the coexistence of functionally distinct classes of CCPs, the knowledge of EAP recruitment hierarchy in between the earliest and latest moments of the CCP life span is still limited. We began to tackle the averaging problem by first grouping CCPs in lifetime cohorts, followed by nonlinear interpolation of average EAP intensity traces within each cohort from alignments relative to the initiation and relative to the termination event (Loerke et al. 2011). More recently, we combined this strategy with master/slave tracking of extremely weak signals (Fig. 5A) (Aguet et al. 2013). As an example, although the endogenous dynamin-2-eGFP signal was not significant except for the last few time points before internalization, when followed at the position of a CCP indicated by the master tdTomato-CLCa channel, it was significantly stronger than the random traces outside CCP images (Fig. 5B). Using averaging of large numbers of dynamin traces in lifetime cohorts confirmed a slow association of dynamin until 20 seconds before termination, in which a sudden spike suggests the assembly of the scission machinery (Fig. 5C, top). Interestingly, this analysis also showed that $\sim 50 \%$ of CCPs have no dynamin recruitment (Fig. 5C, bottom) and that these CCPs terminate along a quasi-exponential single-step decay process indicative of random disassembly at the plasma membrane. In contrast, dynamin-positive CCPs terminate in a process consisting of multiple regulatory steps (Fig. 5D). In terms of methodology, we believe this study sets the stage for an analysis of EAP recruitment to CCPs without introducing artifacts from overexpression of fluorescently tagged EAPs and from averaging over heterotypic patterns that are related to differential EAP functions and variable CCP lifetimes. Thus, the combination of genome editing with multispectral imaging has brought CME imaging in mammalian cells to the same level of molecular accuracy as the yeast model.

\section{CONCLUDING REMARKS AND FUTURE PERSPECTIVES}

What Have We Learned, and What Remains?

To date, probably the most critical lesson learned from imaging $\mathrm{CME}$ is the striking "individualism" of CCPs, especially in mammalian cells (Rappoport and Simon 2003; Ehrlich et al. 2004; Keyel et al. 2004; Merrifield et al. 2005; Loerke et al. 2009; Saffarian et al. 2009; Mettlen et al. 2010; Henry et al. 2012). The heterogeneity in association of cargo and EAPs and lifetimes could not be appreciated in biochemical assays that score ligand uptake by cell ensembles. The physiological relevance and molecular basis of this heterogeneity has yet to be determined. It could be indicative of simply the stochastic nature of CCP assembly from multiple components (Banerjee et al. 2012) or of actual functional differences between CCPs, perhaps owing to different types of cargo. The latter hypothesis is supported by evidence showing that differences in cargo type, size, and loading capacity lead to different recruitment of cargospecific adaptors, which in turn recruit and interact with distinct subsets of EAPs. Thus, cargo-driven differences in the molecular com- 

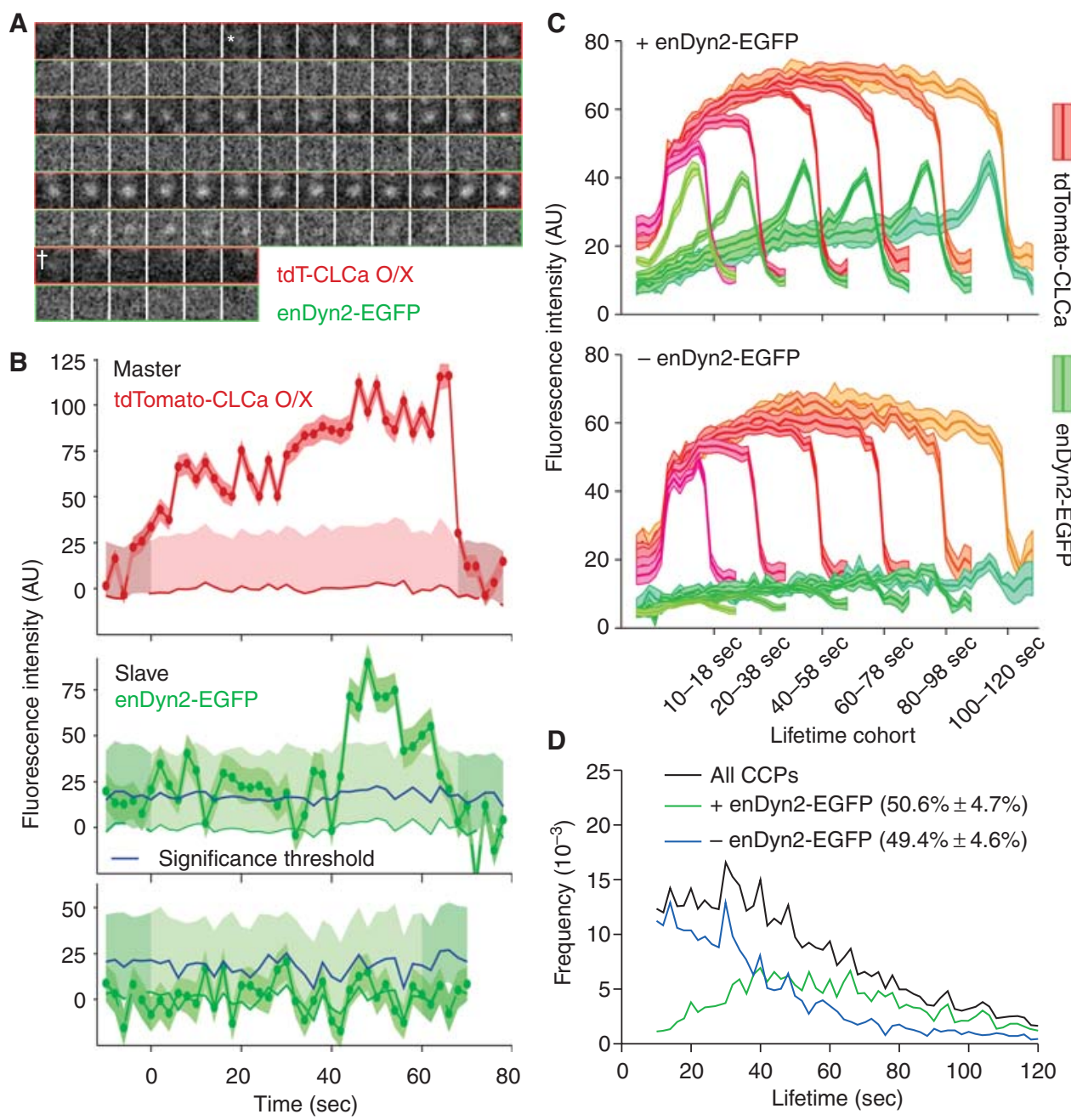

Figure 5. Dynamin is recruited early in CCP formation and is required for CCP maturation (Aguet et al. 2013). (A) Representative images of a CCP visualized in SK-MEL-2 cells overexpressing tdTomato-CLCa (red) and expressing endogenous dynamin-2-EGFP (enDyn2-EGFP, green). (B) Master/slave approach for tracking weak EAP signals. Corresponding intensity traces of tdTomato-CLCa (top; master channel) and enDyn2-EGFP (middle; slave channel), illustrating dynamin recruitment early during CCP formation, followed by a characteristic peak corresponding to assembly of the scission machinery. (Bottom) Representative trace of enDyn2EGFP intensity at a random location outside CCSs. (C) Average clathrin (red tones) and dynamin (green tones) fluorescence intensity traces in lifetime cohorts of Dyn2-positive (top) and Dyn2-negative (bottom) CCPs. Intensities are shown as mean \pm S.E. calculated from eight cells. $(D)$ Lifetime distributions of bona fide CCPs subcategorized as Dyn2-positive (green) or Dyn2-negative (blue).

position of individual CCPs could affect their maturation kinetics (Ehrlich et al. 2004; Veiga and Cossart 2005; Liu et al. 2010; Mettlen et al. 2010; Henry et al. 2012). In addition, spatially confined differences in molecular composition of the plasma membrane, the cytoplasm, or the cortex also affect the kinetics of CCP initiation and maturation (Antonescu et al. 2010; Nunez et al. 2011; Sun and Drubin 2012). On a larger scale, heterogeneity has been described as induced by the mechanical tension in the cortex (Boulant et al. 2011) and by the cellular context 
in multicellular ensembles (Snijder et al. 2009). These results give a first glimpse into how tissue organization and mechanics play fundamental roles in the control of CME dynamics.

Although CCP heterogeneity presents a major challenge for the acquisition of representative data, it offers unique opportunities for dissecting the functional hierarchy among CCP constituents without global perturbation. In concept, the correlation between variation in cargo, adaptors, EAPs, cell location, and lifetime will allow inference of the functional interdependency between these parameters. There are many years to go until such a fluctuation analysis framework will allow comprehensive dissection of the molecular mechanism of CME. It will take further improvements in reproducibility of experiments and image analysis, and implementation of novel statistical methods for multiplexed analysis of component dependencies across experiments. Ultimately, however, it will be the cleanest approach to understand the intricate interactions among the many CCP components.

In parallel to these developments, the scientific community will have to ask how much deeper we need to research the molecular details of CME as opposed to working toward physiologically more realistic experimental models. Recent developments in imaging technology have brought us leaps closer to a quantitative analysis of CME, even at highest resolution, in physiologically relevant ex vivo and in vivo models (we refer to Bökel and Brand 2014 and Weigert 2014 for a discussion of monitoring endocytosis in animal models). Among the most notable advances, soft substrates with high-refraction index allow TIRF imaging in variable mechanical environments (Gutierrez et al. 2011) and light-sheet imaging in combination with structured illumination allows the resolution of near-single-molecule events outside the restricting geometry of an evanescent field (Gao et al. 2012). Light-sheet imaging may also be the key to resolving individual CCP dynamics in cell models inside physiological microenvironments and tissues. Ultimately, the goal of imaging CME in more realistic settings will be to understand endocytosis as a compo- nent process of more complex cell functions such as signaling, migration, or mitosis. Although some studies have begun to address these intersections (Caswell et al. 2009; Sorkin and von Zastrow 2009; Fielding and Royle 2013), it remains the major task for imaging CME to link the spatiotemporal organization of endocytosis in these contexts to the detailed molecular cascades the field has unveiled.

\section{REFERENCES}

\section{${ }^{*}$ Reference is also in this collection.}

Aghamohammadzadeh S, Ayscough KR. 2009. Differential requirements for actin during yeast and mammalian endocytosis. Nat Cell Biol 11: 1039-1042.

Aguet F, Antonescu CN, Mettlen M, Schmid SL, Danuser G. 2013. Advances in analysis of low signal-to-noise images link dynamin and AP2 to the functions of an endocytic checkpoint. Dev Cell 26: 279-291.

Anderson RG, Goldstein JL, Brown MS. 1977. A mutation that impairs the ability of lipoprotein receptors to localise in coated pits on the cell surface of human fibroblasts. Nature 270: 695-699.

Antonescu CN, Danuser G, Schmid SL. 2010. Phosphatidic acid plays a regulatory role in clathrin-mediated endocytosis. Mol Biol Cell 21: 2944-2952.

Banerjee A, Berezhkovskii A, Nossal R. 2012. Stochastic model of clathrin-coated pit assembly. Biophys J 102: 2725-2730.

Bocking T, Aguet F, Harrison SC, Kirchhausen T. 2011. Single-molecule analysis of a molecular disassemblase reveals the mechanism of Hsc70-driven clathrin uncoating. Nat Struct Mol Biol 18: 295-301.

Boettner DR, Chi RJ, Lemmon SK. 2012. Lessons from yeast for clathrin-mediated endocytosis. Nat Cell Biol 14: 2-10.

* Bökel C, Brand M. 2014. Endocytosis and signaling during development. Cold Spring Harb Perspect Biol doi: 10.1101/cshperspect.a017020.

Boulant S, Kural C, Zeeh JC, Ubelmann F, Kirchhausen T. 2011. Actin dynamics counteract membrane tension during clathrin-mediated endocytosis. Nat Cell Biol 13: 1124-1131.

Brady RJ, Damer CK, Heuser JE, O’Halloran TJ. 2010. Regulation of Hip $1 r$ by epsin controls the temporal and spatial coupling of actin filaments to clathrin-coated pits. $J$ Cell Sci 123: 3652-3661.

Burston HE, Maldonado-Baez L, Davey M, Montpetit B, Schluter C, Wendland B, Conibear E. 2009. Regulators of yeast endocytosis identified by systematic quantitative analysis. J Cell Biol 185: 1097-1110.

Carroll SY, Stirling PC, Stimpson HE, Giesselmann E, Schmitt MJ, Drubin DG. 2009. A yeast killer toxin screen provides insights into A/B toxin entry, trafficking, and killing mechanisms. Dev Cell 17: 552-560.

Carroll SY, Stimpson HE, Weinberg J, Toret CP, Sun Y, Drubin DG. 2012. Analysis of yeast endocytic site formation 
M. Mettlen and G. Danuser

and maturation through a regulatory transition point Mol Biol Cell 23: 657-668.

Caswell PT, Vadrevu S, Norman JC. 2009. Integrins: Masters and slaves of endocytic transport. Nat Rev Mol Cell Biol 10: $843-853$.

Cocucci E, Aguet F, Boulant S, Kirchhausen T. 2012. The first five seconds in the life of a clathrin-coated pit. Cell 150: 495-507.

Conner SD, Schmid SL. 2003. Regulated portals of entry into the cell. Nature 422: 37-44.

Danuser G. 2011. Computer vision in cell biology. Cell 147: 973-978.

Doyon JB, Zeitler B, Cheng J, Cheng AT, Cherone JM, Santiago Y, Lee AH, Vo TD, Doyon Y, Miller JC, et al. 2011. Rapid and efficient clathrin-mediated endocytosis revealed in genome-edited mammalian cells. Nat Cell Biol 13: 331-337.

Ehrlich M, Boll W, Van Oijen A, Hariharan R, Chandran K, Nibert ML, Kirchhausen T. 2004. Endocytosis by random initiation and stabilization of clathrin-coated pits. Cell 118: 591-605.

Engqvist-Goldstein AE, Drubin DG. 2003. Actin assembly and endocytosis: From yeast to mammals. Annu Rev Cell Dev Biol 19: 287-332.

Fielding AB, Royle SJ. 2013. Mitotic inhibition of clathrinmediated endocytosis. Cell Mol Life Sci 70: 3423-3433.

Gaidarov I, Santini F, Warren RA, Keen JH. 1999. Spatial control of coated-pit dynamics in living cells. Nat Cell Biol 1: 1-7.

Gao L, Shao L, Higgins CD, Poulton JS, Peifer M, Davidson MW, Wu X, Goldstein B, Betzig E. 2012. Noninvasive imaging beyond the diffraction limit of 3D dynamics in thickly fluorescent specimens. Cell 151: 1370-1385.

Gutierrez E, Tkachenko E, Besser A, Sundd P, Ley K, Danuser G, Ginsberg MH, Groisman A. 2011. High refractive index silicone gels for simultaneous total internal reflection fluorescence and traction force microscopy of adherent cells. PLoS ONE 6: e23807.

Henry AG, Hislop JN, Grove J, Thorn K, Marsh M, von Zastrow M. 2012. Regulation of endocytic clathrin dynamics by cargo ubiquitination. Dev Cell 23: 519-532.

Huang KM, D’Hondt K, Riezman H, Lemmon SK. 1999. Clathrin functions in the absence of heterotetrameric adaptors and AP180-related proteins in yeast. EMBO J 18: $3897-3908$.

Huckaba TM, Gay AC, Pantalena LF, Yang HC, Pon LA. 2004. Live cell imaging of the assembly, disassembly, and actin cable-dependent movement of endosomes and actin patches in the budding yeast, Saccharomyces cerevisiae. J Cell Biol 167: 519-530.

Jaqaman K, Loerke D, Mettlen M, Kuwata H, Grinstein S, Schmid SL, Danuser G. 2008. Robust single-particle tracking in live-cell time-lapse sequences. Nat Methods 5: 695-702.

Jaqaman K, Kuwata H, Touret N, Collins R, Trimble WS, Danuser G, Grinstein S. 2011. Cytoskeletal control of CD36 diffusion promotes its receptor and signaling function. Cell 146: 593-606.

Kaksonen M, Sun Y, Drubin DG. 2003. A pathway for association of receptors, adaptors, and actin during endocytic internalization. Cell 115: 475-487.
Kaksonen M, Toret CP, Drubin DG. 2005. A modular design for the clathrin- and actin-mediated endocytosis machinery. Cell 123: 305-320.

Kaksonen M, Toret CP, Drubin DG. 2006. Harnessing actin dynamics for clathrin-mediated endocytosis. Nat Rev Mol Cell Biol 7: 404-414.

Keyel PA, Watkins SC, Traub LM. 2004. Endocytic adaptor molecules reveal an endosomal population of clathrin by total internal reflection fluorescence microscopy. J Biol Chem 279: 13190-13204.

Liu AP, Aguet F, Danuser G, Schmid SL. 2010. Local clustering of transferrin receptors promotes clathrin-coated pit initiation. J Cell Biol 191: 1381-1393.

Loerke D, Mettlen M, Yarar D, Jaqaman K, Jaqaman H, Danuser G, Schmid SL. 2009. Cargo and dynamin regulate clathrin-coated pit maturation. PLoS Biol 7: e57.

Loerke D, Mettlen M, Schmid SL, Danuser G. 2011. Measuring the hierarchy of molecular events during clathrinmediated endocytosis. Traffic 12: 815-825.

Macro L, Jaiswal JK, Simon SM. 2012. Dynamics of clathrinmediated endocytosis and its requirement for organelle biogenesis in Dictyostelium. J Cell Sci 125: 5721-5732.

Maddox PS, Moree B, Canman JC, Salmon ED. 2003. Spinning disk confocal microscope system for rapid high-resolution, multimode, fluorescence speckle microscopy and green fluorescent protein imaging in living cells. Methods Enzymol 360: 597-617.

Massol RH, Boll W, Griffin AM, Kirchhausen T. 2006. A burst of auxilin recruitment determines the onset of clathrin-coated vesicle uncoating. Proc Natl Acad Sci 103: 10265-10270.

Mattheyses AL, Atkinson CE, Simon SM. 2011. Imaging single endocytic events reveals diversity in clathrin, dynamin and vesicle dynamics. Traffic 12: 1394-1406.

McMahon HT, Boucrot E. 2011. Molecular mechanism and physiological functions of clathrin-mediated endocytosis. Nat Rev Mol Cell Biol 12: 517-533.

Meijering E, Dzyubachyk O, Smal I. 2012. Methods for cell and particle tracking. Methods Enzymol 504: 183-200.

* Merrifield C, Kaksonen M. 2014. Endocytic accessory factors and regulation of clathrin-mediated endocytosis. Cold Spring Harb Perspect Biol doi: 10.1101/cshperspect.a016733.

Merrifield CJ, Perrais D, Zenisek D. 2005. Coupling between clathrin-coated-pit invagination, cortactin recruitment, and membrane scission observed in live cells. Cell 121: 593-606.

Mettlen M, Stoeber M, Loerke D, Antonescu CN, Danuser G, Schmid SL. 2009. Endocytic accessory proteins are functionally distinguished by their differential effects on the maturation of clathrin-coated pits. Mol Biol Cell 20: 3251-3260.

Mettlen M, Loerke D, Yarar D, Danuser G, Schmid SL. 2010. Cargo- and adaptor-specific mechanisms regulate clathrin-mediated endocytosis. J Cell Biol 188: 919-933.

Mitsunari T, Nakatsu F, Shioda N, Love PE, Grinberg A, Bonifacino JS, Ohno H. 2005. Clathrin adaptor AP-2 is essential for early embryonal development. Mol Cell Biol 25: 9318-9323.

Mooren OL, Galletta BJ, Cooper JA. 2012. Roles for actin assembly in endocytosis. Annu Rev Biochem 81: 661-686. 
Dynamics of Clathrin-Mediated Endocytosis

Newpher TM, Smith RP, Lemmon V, Lemmon SK. 2005. In vivo dynamics of clathrin and its adaptor-dependent recruitment to the actin-based endocytic machinery in yeast. Dev Cell 9: 87-98.

Nunez D, Antonescu C, Mettlen M, Liu A, Schmid SL, Loerke D, Danuser G. 2011. Hotspots organize clathrin-mediated endocytosis by efficient recruitment and retention of nucleating resources. Traffic 12: $1868-1878$.

O'Halloran TJ, Anderson RG. 1992. Clathrin heavy chain is required for pinocytosis, the presence of large vacuoles, and development in Dictyostelium. J Cell Biol 118: 13711377.

Pampaloni F, Stelzer EH, Leicht S, Marcello M. 2010. Madin-Darby canine kidney cells are increased in aerobic glycolysis when cultured on flat and stiff collagen-coated surfaces rather than in physiological 3-D cultures. Proteomics 10: 3394-3413.

Pearse BM. 1975. Coated vesicles from pig brain: Purification and biochemical characterization. J Mol Biol 97: 9398.

Pearse BM. 1976. Clathrin: A unique protein associated with intracellular transfer of membrane by coated vesicles. Proc Natl Acad Sci 73: 1255-1259.

Pearse BM. 1978. On the structural and functional components of coated vesicles. J Mol Biol 126: 803-812.

Rappoport JZ, Simon SM. 2003. Real-time analysis of clathrin-mediated endocytosis during cell migration. J Cell Sci 116: 847-855.

Rink J, Ghigo E, Kalaidzidis Y, Zerial M. 2005. Rab conversion as a mechanism of progression from early to late endosomes. Cell 122: 735-749.

Ruscetti T, Cardelli JA, Niswonger ML, O’Halloran TJ. 1994. Clathrin heavy chain functions in sorting and secretion of lysosomal enzymes in Dictyostelium discoideum. J Cell Biol 126: 343-352.

Saffarian S, Cocucci E, Kirchhausen T. 2009. Distinct dynamics of endocytic clathrin-coated pits and coated plaques. PLoS Biol 7: e1000191.

Sbalzarini IF, Koumoutsakos P. 2005. Feature point tracking and trajectory analysis for video imaging in cell biology. $J$ Struct Biol 151: 182-195.
Schmid EM, McMahon HT. 2007. Integrating molecular and network biology to decode endocytosis. Nature 448: $883-888$.

Schwarz G. 1978. Estimating dimension of a model. Ann Stat 6: 461-464.

Sirotkin V, Beltzner CC, Marchand JB, Pollard TD. 2005. Interactions of WASp, myosin-I, and verprolin with Arp2/3 complex during actin patch assembly in fission yeast. J Cell Biol 170: 637-648.

Sirotkin V, Berro J, Macmillan K, Zhao L, Pollard TD. 2010. Quantitative analysis of the mechanism of endocytic actin patch assembly and disassembly in fission yeast. $\mathrm{Mol}$ Biol Cell 21: 2894-2904.

Smaczynska-de R II, Allwood EG, Aghamohammadzadeh S, Hettema EH, Goldberg MW, Ayscough KR. 2010. A role for the dynamin-like protein Vps1 during endocytosis in yeast. J Cell Sci 123: 3496-3506.

Snijder B, Sacher R, Ramo P, Damm EM, Liberali P, Pelkmans L. 2009. Population context determines cell-to-cell variability in endocytosis and virus infection. Nature 461: $520-523$.

Sorkin A, von Zastrow M. 2009. Endocytosis and signalling: Intertwining molecular networks. Nat Rev Mol Cell Bio 10: 609-622.

Sun Y, Drubin DG. 2012. The functions of anionic phospholipids during clathrin-mediated endocytosis site initiation and vesicle formation. J Cell Sci 125: 6157-6165.

Tan PK, Davis NG, Sprague GF, Payne GS. 1993. Clathrin facilitates the internalization of seven transmembrane segment receptors for mating pheromones in yeast. $J$ Cell Biol 123: $1707-1716$.

Taylor MJ, Perrais D, Merrifield CJ. 2011. A high precision survey of the molecular dynamics of mammalian clathrin-mediated endocytosis. PLoS Biol 9: e1000604.

Toomre D, Manstein DJ. 2001. Lighting up the cell surface with evanescent wave microscopy. Trends Cell Biol 11: 298-303.

Veiga E, Cossart P. 2005. Listeria hijacks the clathrin-dependent endocytic machinery to invade mammalian cells. Nat Cell Biol 7: 894-900.

* Weigert R. 2014. Imaging the dynamics of endocytosis in live mammalian tissues. Cold Spring Harb Perspect Biol doi: 10.1101/cshperspect.a017012. 


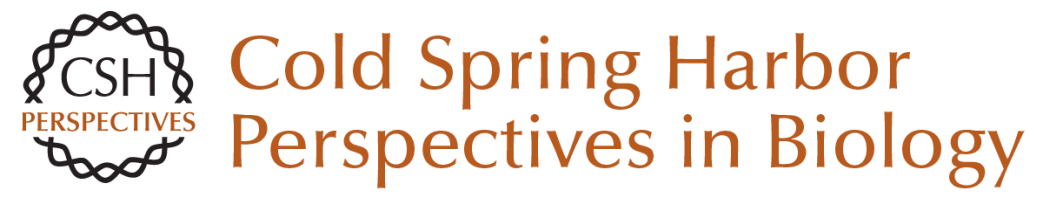

\section{Imaging and Modeling the Dynamics of Clathrin-Mediated Endocytosis}

Marcel Mettlen and Gaudenz Danuser

Cold Spring Harb Perspect Biol 2014; doi: 10.1101/cshperspect.a017038 originally published online August 28, 2014

\section{Subject Collection Endocytosis}

Endocytosis: Past, Present, and Future Sandra L. Schmid, Alexander Sorkin and Marino Zerial

Rab Proteins and the Compartmentalization of the Endosomal System Angela Wandinger-Ness and Marino Zerial

Cargo Sorting in the Endocytic Pathway: A Key Regulator of Cell Polarity and Tissue Dynamics Suzanne Eaton and Fernando Martin-Belmonte

Unconventional Functions for Clathrin, ESCRTs, and Other Endocytic Regulators in the

Cytoskeleton, Cell Cycle, Nucleus, and Beyond:

Links to Human Disease Frances M. Brodsky, R. Thomas Sosa, Joel A. Ybe, et al.

Endocytosis of Viruses and Bacteria Pascale Cossart and Ari Helenius

Lysosomal Adaptation: How the Lysosome

Responds to External Cues Carmine Settembre and Andrea Ballabio

Reciprocal Regulation of Endocytosis and Metabolism

Costin N. Antonescu, Timothy E. McGraw and Amira Klip

Endocytosis and Autophagy: Exploitation or Cooperation?

Sharon A. Tooze, Adi Abada and Zvulun Elazar
Imaging and Modeling the Dynamics of Clathrin-Mediated Endocytosis Marcel Mettlen and Gaudenz Danuser

Endocytic Accessory Factors and Regulation of Clathrin-Mediated Endocytosis Christien J. Merrifield and Marko Kaksonen

The Complex Ultrastructure of the Endolysosomal

System Judith Klumperman and Graça Raposo

The Biogenesis of Lysosomes and

Lysosome-Related Organelles J. Paul Luzio, Yvonne Hackmann, Nele M.G. Dieckmann, et al.

Endocytosis, Signaling, and Beyond Pier Paolo Di Fiore and Mark von Zastrow

Clathrin-Independent Pathways of Endocytosis Satyajit Mayor, Robert G. Parton and Julie G. Donaldson

The Role of Endocytosis during Morphogenetic Signaling Marcos Gonzalez-Gaitan and Frank Jülicher

Role of Endosomes and Lysosomes in Human Disease Frederick R. Maxfield

For additional articles in this collection, see http://cshperspectives.cshlp.org/cgi/collection/

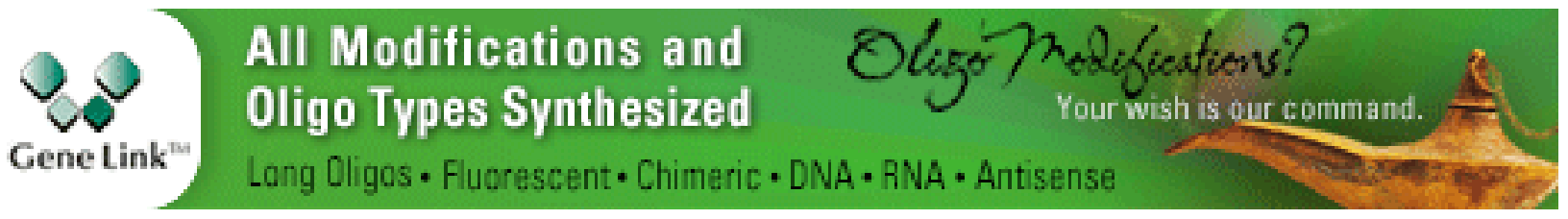


For additional articles in this collection, see http://cshperspectives.cshlp.org/cgi/collection/

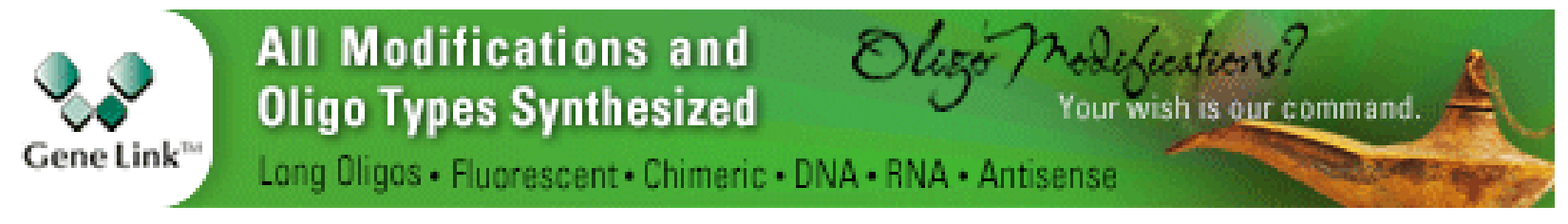

Copyright @ 2014 Cold Spring Harbor Laboratory Press; all rights reserved 